\title{
Acute lower limb ischemia following Angio-Seal deployment after transfemoral percutaneous coronary intervention
}

\author{
Michał Walczewski, Adam Rdzanek, Ewa Pędzich-Placha, Janusz Kochman \\ $1^{\text {st }}$ Department of Cardiology, Medical University of Warsaw, Warsaw, Poland
}

Kardiochirurgia i Torakochirurgia Polska 2019; 16 (2): 103-105

Vascular closure devices (VCDs) have shown effectiveness in randomized trials and have been in clinical use for many years [1]. Their main advantage is in achieving complete hemostasis as effectively as classic manual compression, but within a significantly shorter period of time [2]. Taking into consideration hospital economics and patient comfort, the use of VCDs is also associated with a shorter immobilization period [2]. It is especially important in centers treating high numbers of patients and where patients are discharged shortly after a percutaneous procedure. However, some meta-analyses have raised doubts about the superiority of VCDs over manual compression (MC) in the case of serious complications [3]. We present a case of a patient with VCD complications, followed by a short literature review.

A 91-year-old woman with type 2 diabetes, chronic kidney disease, anemia, hypertension and peripheral artery disease (PAD) was admitted to hospital due to experiencing recurrent retrosternal pain for several days. An ECG showed ST segment depressions in lateral wall leads and elevated cardiac troponin levels.

She underwent urgent coronary angiography followed by angioplasty with zotarolimus-eluting stent implantation. The procedure was performed from the femoral approach with a good hemodynamic result. An Angio-Seal device was used to close the access site. The closure procedure was performed step-by-step, but complete hemostasis was not achieved. Due to active minor bleeding, manual compression and a pressure bandage were applied. After the procedure, the patient was in a good clinical condition and was observed overnight. Twelve hours of bed rest was recommended. The next morning, the patient reported a sudden loss of movement and numbness in the right foot. In the clinical examination, the right lower limb was cold and pale below the knee joint. The pulse was slightly palpable on the femoral artery and not palpable on the popliteal artery. Due to symptoms of acute limb ischemia, a computed tomography (CT) scan was performed. It revealed a critical occlusion in the right common femoral artery and relevant atherosclerotic stenosis in the distal part of the vessel (Fig. 1).

The patient underwent emergency thrombectomy. The surgeon found an intravascular component of the AngioSeal blocking the deep femoral artery (Fig. 2). It was suc-

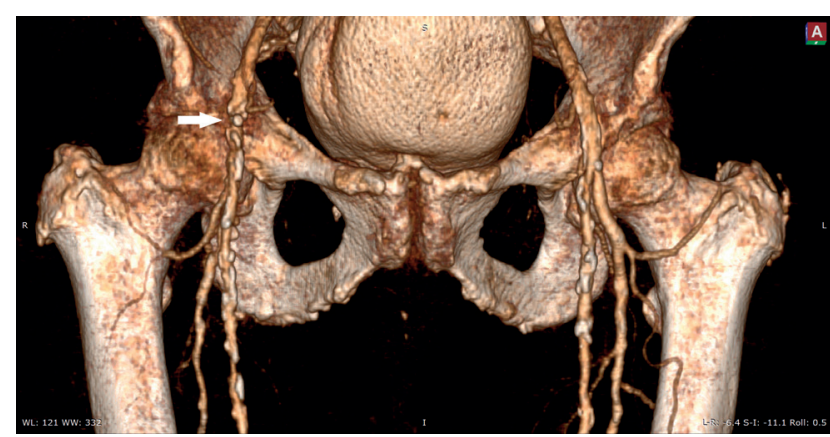

Fig. 1. 3D reconstruction of the blocked artery

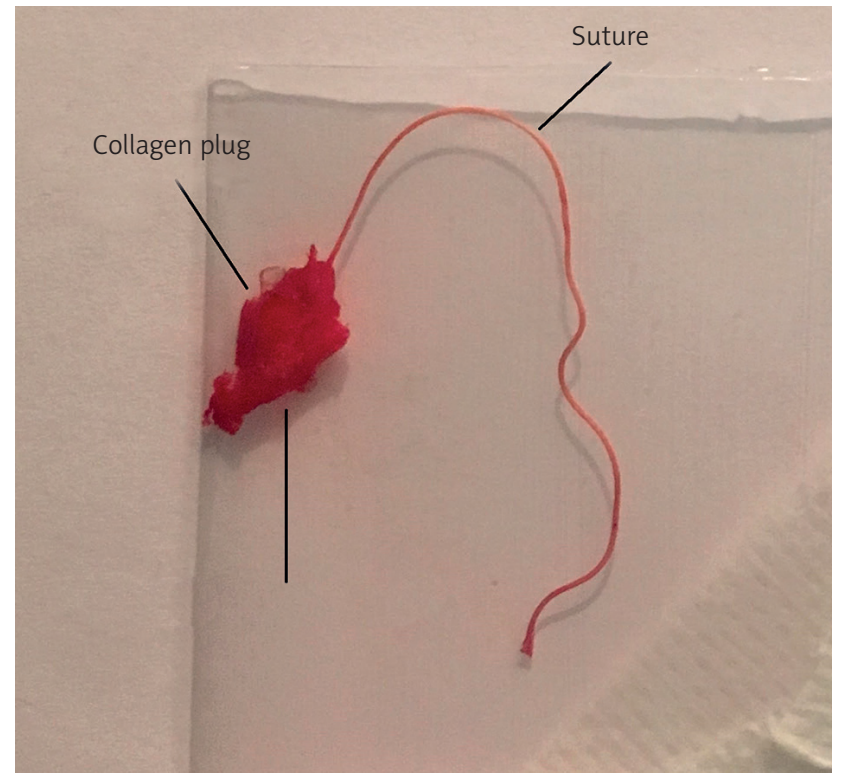

Fig. 2. Angio-Seal removed by the surgeon

Address for correspondence: Michat Walczewski, $1^{\text {st }}$ Department of Cardiology, Medical University of Warsaw, 1 a Banacha St, 02-097 Warsaw, Poland, e-mail: walcz.michal@gmail.com

Received: 18.02.2019, accepted: 29.04.2019. 
cessfully removed and blood flow was restored. After surgery, signs of ischemia disappeared and the patient was discharged a few days later in a good clinical condition.

There are three main mechanisms of action used in closure devices: clip-, collagen- or suture-based. Our patient was treated with an Angio-Seal device. In these particular VCDs, the bioabsorbable anchor is positioned intraluminally to seal the vessel from the inside. The collagen plug clogs the artery from the outside. The anchor and plug are pulled together closely by a suture. In the case of our patient, this whole complex penetrated to the lumen of the vessel. It resulted in vascular obstruction and limb ischemia requiring surgical intervention. These rare complications are associated mainly with closure devices. A previous meta-analysis published in the American Heart Journal raised awareness about the safety of VCDs regarding these serious adverse events [3]. Surprisingly, investigators in the latest Cochrane Systematic Review focused on closure devices found no evidence of vascular injury requiring repair when comparing manual compression with VCDs, even though studies measuring limb ischemia as an outcome were included [4]. This effect was observed regardless of the mechanism of action of VCDs. The majority of the studies did not report any cases of limb ischemia or vascular obstruction requiring surgical intervention. More common adverse events affect the puncture site. Data from available studies show that the frequency of those minor complications is similar in closure device and manual compression patients. Overall, the incidence of complications varies between $2.9 \%$ and $3.8 \%$ in different study groups $[5,6]$. The complications are mainly bleeding, hematoma and pseudoaneurysms. These results are supported by a recent meta-analysis [3]. Some meta-analyses and studies indicate a significantly lower incidence of hematomas in VCD treated patients [1]. The latest Cochrane Systematic Review, which included 52 studies (19,192 participants), notes that this effect is dependent on the mechanism of action of VCDs and was observed only in collagen-based devices when compared to manual compression [4]. The authors concluded that the incidence of pseudoaneurysms was also lower in patients treated with collagen-based VCDs. However, when comparing other types of closure devices with extrinsic compression, no significant differences in the frequency of complications were observed.

The use of Angio-Seal on a frail patient with concomitant renal failure, as presented in our case, was justified by the intention of achieving hemostasis quickly in order to avoid blood loss and possible complications. Most studies indicate a notably shorter time to hemostasis with the use of closure devices. In the Cochrane Systematic Review, the authors conclude that the analyzed studies are too heterogeneous to be combined. Nonetheless, both metal clip-based and suture-based VCDs were associated with reduced time to hemostasis when compared with extrinsic compression [4].

The patient in our case presented many of the predictors of VCD-related vascular complications listed below, including older age, female sex, diabetes, long procedure time, renal failure, PAD, etc. It is worth mentioning that precautions were taken and the patient was ordered to rest in bed for up to $12 \mathrm{~h}$, yet despite this, an adverse event occurred. It would be beneficial to determine the cause of intraluminal dislocation of the device. According to other authors we assume that probable causes were inadequate pressure applied to the suture during deployment or placement of the VCD over the atherosclerotic plaque [7]. Both of these scenarios could lead to vessel wall rupture and dislodgment of the device to the distal part of the artery, resulting in limb ischemia. However, mechanisms of VCD insertion failure are not well described and available studies are generally focused on finding risk factors of vascular complications.

Several studies have been performed to establish predictors ofVCDs adverse events $[1,5,6]$. One of the studies pointed out certain risk factors, including age greater than 70 years $(\mathrm{OR}=10.29 ; p=0.04)$, diabetes mellitus $(\mathrm{DM})(\mathrm{OR}=1.39$; $p=0.02)$, total number of comorbidities $(\mathrm{OR}=2.73 ; p=0.04)$, duration of the procedure $(\mathrm{OR}=1.02 ; p=0.03)$, and number of stents placed $(\mathrm{OR}=3.05 ; p=0.04)$. However, after propensity score adjustment, only age $>70$ was a significant predictive factor in multivariate analysis, and increased the risk of complications tenfold [5]. A study by Holm et al., which included 1,001 patients, identified female gender and multiple punctures as independent predictors of adverse events and large hematomas [1]. Dumont et al. performed a retrospective analysis of 11,119 patients who underwent cardiac catheterization and/or percutaneous intervention, with femoral artery access, regardless of the use of closure devices [6]. Increased risk for vascular complications was found in patients who were older than 70 years, were female, had renal failure, underwent percutaneous intervention, and had a venous sheath. In the literature both female gender and older age are correlated with risk of vascular complications.

Proper imaging is also vital regarding safe VCD placement. Instructions for use by the manufacturer emphasize that the angiogram of the puncture site is mandatory prior to placing Angio-Seal and in the case of PAD the device can be deployed safely in patient arteries $>5 \mathrm{~mm}$ diameter when there is found to be no luminal narrowing of $40 \%$ or greater within $5 \mathrm{~mm}$ of the puncture site. Moreover, some retrospective studies demonstrate that ultrasound-guided insertion of VCDs can reduce the risk of all complications, although there is no evidence for the routine use of this method [8].

In conclusion, we have presented a case of a rare serious adverse event in a patient who received a vascular closure device after a percutaneous procedure. Our patient presented multiple risk factors of vascular complications. We must emphasize that the evidence available in the literature does not show an increased incidence of serious complications or higher mortality rate in patients treated with VCDs. Other data indicate a decreased rate of minor and local complications with the use of collagen-based devices. 


\section{Disclosure}

The authors report no conflict of interest.

\section{References}

1. Holm NR, Sindberg B, Schou M, Maeng M, Kaltoft A, Bottcher M, Krusell LR, Hjort J, Thuesen L, Terkelsen CJ, Christiansen EH, Bøtker HE, Kristensen SD, Lassen JF; CLOSE-UP study group. Randomised comparison of manual compression and FemoSeal vascular closure device for closure after femoral artery access coronary angiography: the CLOSure dEvices Used in everyday Practice (CLOSE-UP) study. Eurolntervention 2014; 10: 183-190.

2. Wong HF, Lee CW, Chen YL, Wu YM, Weng HH, Wang YH, Liu HM. Prospective comparison of angio-seal versus manual compression for hemostasis after neurointerventional procedures under systemic heparinization. Am J Neuroradiol 2013; 34: 397-401

3. Biancari F, D'Andrea V, Di Marco C, Savino G, Tiozzo V, Catania A. Meta-analysis of randomized trials on the efficacy of vascular closure devices after diagnostic angiography and angioplasty. Am Heart J 2010; 159: 518-531.
4. Robertson L, Andras A, Colgan F, Jackson R. Vascular closure devices for femoral arterial puncture site haemostasis. Cochrane Database Syst Rev 2016; 3 Cd009541.

5. Wu PJ, Dai YT, Kao HL, Chang CH, Lou MF. Access site complications following transfemoral coronary procedures: comparison between traditional compression and angioseal vascular closure devices for haemostasis. BMC Cardiovasc Disord 2015; 15: 34.

6. Dumont CJ, Keeling AW, Bourguignon C, Sarembock IJ, Turner M. Predictors of vascular complications post diagnostic cardiac catheterization and percutaneous coronary interventions. Dimens Crit Care Nurs 2006; 25: 137-142.

7. Lasam G, Oaks JB, Brensilver J. Angio-SealTM embolization: a rare etiology of an acute distal limb ischemia. Case Rep Vasc Med 2017; 2017: 9613863.

8. Lucatelli P, Cannavale A, Cirelli C, d'Adamo A, Salvatori FM, Fanelli F. Use of ultrasound in the insertion of a vascular closure device: a comparative retrospec tive study with the standard blind technique. Radiol Med 2015; 120: 283-288. 